\title{
Randomized placebo-controlled cross-over designs in clinical trials: A gold-standard to be reassessed
}

\author{
Guillaume Leonard, Ph.D. ${ }^{1}$, Sylvie Lafrenaye, M.D. ${ }^{2}$, Philippe Goffaux, Ph.D. ${ }^{3 *}$
}

\author{
${ }^{1}$ School of Rehabilitation \\ Université de Sherbrooke, Faculty of Medicine, \\ Sherbrooke, Québec, Canada, J1H 5N4. \\ ${ }^{2}$ Departement of Pediatrics \\ Université de Sherbrooke, Faculty of Medicine, \\ Sherbrooke, Québec, Canada, J1H 5N4. \\ ${ }^{3}$ Department of Surgery - Neurosurgery Service, \\ Université de Sherbrooke, Faculty of Medicine, \\ Sherbrooke, Québec, Canada, J1H 5N4.
}

Pages : 10

Tables : 1

Figures : 0

*Corresponding author: Philippe Goffaux, Ph.D.

Université de Sherbrooke

Faculty of Medicine, Department of Neurosurgery-Neurosurgery Service $3001,12^{\mathrm{e}}$ avenue nord

Sherbrooke (Québec) Canada J1H 5N4 


\begin{abstract}
Placebo effects are well-known phenomena in medicine and biology. In fact, placebos are used as control conditions in randomized cross-over clinical trials to validate new treatments. Only recently, however, has it become apparent that the conditioning and/or expectation effects provided by the experience of placebos can influence the results of clinical trials. It seems that combining shams and sequences has prejudiced the conclusions provided by cross-over designs. Frighteningly, this bias is always in the same direction, namely to increase the risk of rejecting potentially valid treatments. New models for clinical trials should be encouraged if we wish to market new and truly valid treatments.
\end{abstract}


Are placebo-controlled cross-over designs in clinical trials silently killing new and promising treatments? Raising this question could seem heretic, especially in an age where evidence-based medicine and bias-prevention constitute omnipresent concerns. Sadly, this may be happening every day, hiding in plain view of our collective ignorance. The proverbial fly in the ointment seems to be in the nature of the design itself - a design which exposes participants to multiple interventions, often in close succession. Although multiple treatment expositions offer several recognized advantages (e.g., preventing the prejudicial effects provided by inter-individual differences and decreasing the number of participants needed to obtain appropriate statistical power), they also promote unsuspected carry-over effects which can negatively influence the confirmation of new treatments. Cross-over designs, therefore, may be designed to favor bias.

\section{The problem: Placebo effects carry consequences}

This tendency to favor bias was made obvious in a recent study published by Andre-Obadia and colleagues ${ }^{1}$. In their study, the authors used a double-blind, randomized, cross-over design to investigate the analgesic effects of repetitive transcranial magnetic stimulation (rTMS), and found that posteroanterior rTMS was more effective than sham and/or lateromedial rTMS. Interestingly, the authors observed that the analgesic effects induced by posteroanterior rTMS were easier to demonstrate when compared with lateromedial rTMS than when compared with sham-rTMS. This observation led Andre-Obadia et al. to conduct a second study in which they specifically evaluated sequence effects (i.e., pertaining to the timing of the different interventions within the protocol) $)^{2}$. They found that the sham intervention significantly decreased pain when it followed a successful rTMS session. On the other hand, there was a tendency for sham-rTMS to increase pain when it followed an unsuccessful rTMS session. Only when the sham intervention was applied first did it provide scores that were 
comparable to baseline (i.e., no intervention at all). Taken together, these observations indicate that the efficacy of past treatments can have a major impact on the efficacy of future treatments, even when these future treatments are designed as inactive controls. This means that placebo interventions may not be, and perhaps have never been, valid control conditions in randomized crossover trials.

From a theoretical perspective, the observations of Andre-Obadia et al. ${ }^{2}$ can be explained by the conditioning model of placebo analgesia ${ }^{3}$. According to classical conditioning theory, the repeated experience of an effective analgesic treatment (the unconditioned stimulus) facilitates the development of robust associations between active treatment effects (in this case active rTMS-induced analgesia) and the surrounding environment (laboratory, rTMS coil, experimenter, etc.). Once the conditioning phase is over, simply presenting the neutral stimulus can produce the unconditioned response, even in the absence of active ingredients. Although this issue is still debated in the field, it is generally admitted that the conditioning effect for pain and other conscious processes relies heavily on expectations created during the conditioning phase $\mathrm{4}^{4}$. A few reviews address the influence of expectations, conditioning, and, other confirmatory phenomena in placebo studies ${ }^{3,5-7}$. However, regardless of the variables which may affect placebo and placebo-like phenomena, it is important to point out that, in clinical trials, pre-crossover conditions may very well produce conditioned responses which can affect post-crossover conditions.

Of course, conditioned responses are not limited to placebo or sham conditions, but may also affect active treatments. Thus, given the right circumstance, it may be possible to kill the efficacy of valid treatments. We recently observed this phenomenon in a study designed to investigate the involvement of endogenous opioids in high-frequency TENS analgesia ${ }^{8}$. Specifically, we found that when TENS was first experienced as ineffective (because of opioid blockade with naloxone) the analgesic effect of all subsequent TENS treatments were completely blocked, even though naloxone was never again provided. Had sequence effects been ignored, we would have erroneously concluded 
that high-frequencey TENS was not opioidergic. In our study participants never experienced TENS prior to their participation. This is worth mentioning because prior experience of the treatment at hand might have influenced treatment efficacy during the first condition, and thus, might have further influenced carry-over effects. Given this possibility, it is always a good idea to take prior treatment experiences into consideration before conducting clinical trials.

The study of Andre-Obadia et al. ${ }^{2}$ and our study ${ }^{8}$ represent opposite sides of the same conditioning coin. On the one hand, the study of Andre-Obadia et al. illustrates that an effective treatment can have positive carry-over effects that can render inert treatments effective, and, on the other hand, our study shows that an ineffective treatment can have negative carry-over effects that can render active treatments ineffective*. These findings suggest that even under stable conditions and even in the absence of important disease-induced fluctuations (two important prerequisites when conducting cross-over designs $)^{9,10}$, the results obtained from cross-over designs need to be interpreted cautiously, and perhaps even questioned outright. In particular, the present studies constitute eloquent examples of why crossover designs run the risk of providing false negatives, either because placebo controls become active and thus hard to beat or because active treatments are rendered completely ineffective. We wish to point out here that it may still be possible for new treatments to survive the biasing effects inherent to cross-over designs, and thus outperform shams. When this happens, researchers can be quite sure that the new treatment they are investigating is truly potent. Unfortunately, short of being extremely potent, many new (and effective) drugs may not survive the biasing effects of carry-over, and thus be cavalierly dismissed.

\footnotetext{
* Treatment failure in our study was caused by the use of naloxone, which provoked a strong abolition of analgesia. On the other hand, treatment failure in conventional clinical trials is typically caused by the use of an inactive substance (i.e., a placebo), which likely provokes a milder degree of treatment failure. As a result, researchers can probably expect weaker negative carry-over effects than ours when running conventional clinical trials. Nevertheless, negative carry-over effects remain a very real possibility; even in placebo-controlled cross-over trials.
} 


\section{Decaying treatment effects may be the solution}

In their paper, Andre-Obadia et al. ${ }^{2}$ suggested two solutions to try and correct for conditioning effects. The first solution proposed by the authors was to dismiss the use of placebos in cross-over designs and use only active interventions. In our opinion, this strategy does not address the problem since the experience made during the first intervention can still influence the response to the second intervention, even if the second intervention is an active treatment. Hence, comparisons made only between active treatments are not the solution to the ill-effects of crossover designs. The second solution proposed by the authors is to change the random nature of presentation by specifically favoring placebos as the first condition. This would have the advantage of preventing positive carryover effects stemming from the initial experience of active treatments. However, this strategy will not correct for the negative carry-over effects stemming from the initial experience of placebo interventions, and thus, again, does not constitute a valid solution to the conditioning problem.

We propose an alternative solution, based on the observations made a number of years ago by Fedele and colleagues ${ }^{11}$. In their study, the authors noted a positive treatment effect during placebo trials which, interestingly, disappeared progressively following repeat exposures to placebos ${ }^{11,12}$. Sadly, the results published by Fedele et al. have languished in obscurity, and researchers have failed to exploit the progressive decline in placebo efficacy observed by the authors. Building on Fedele et al.'s results, it is possible to propose a new approach to clinical research trials, one which would follow treatments over time, as described in Table 1. This would allow researchers (1) to document and study resistance to extinction, a property of active but not placebo treatments, and, (2) to identify the appropriate time point to use when comparing active and placebo treatments.

Evidently, the solution we propose has the disadvantage of increasing the cost and duration of clinical trials. We nevertheless believe this to be necessary in order to ensure the validity of results obtained from cross-over designs. The time needed for conditioning and/or expectation effects to 
extinguish remains uncertain, and likely to vary as a function of conditioned effects. Clearly, future studies are needed to evaluate the time needed for conditioning and/or expectation effects to extinguish, and, to study the impact this may have on the running of clinical trials.

\section{Conclusion}

In conclusion, the study of Andre-Obadia et al. ${ }^{2}$ and the study of Leonard et al. ${ }^{8}$ illustrate that the results of cross-over designs need to be interpreted cautiously. The reader must also acknowledge that sequence effects do not only affect pain research. Indeed, placebo effects are observed in a wide variety of other conditions and treatments ${ }^{13}$, making it probable that sequence effects are also present in cross-over designs aimed at evaluating the efficacy of treatments in other health conditions. The use of cross-over designs that include measurements at multiple time points represent, in our view, a viable solution for researchers who wish to continue using cross-over designs, but who do not want to run the risk of erroneously shelving promising new treatments. The field is ready for novel research studies and targeted review papers on this potentially influential issue. 


\section{Transparency}

\section{Declaration of funding}

The authors have received no payment in preparation of this manuscript

\section{Declaration of financial/other relationships}

G.L., S.L., and, P.G. have disclosed that they have no significant relationships with or financial interests in any commercial companies related to this study or article. 
Table 1. Repeated measures strategy proposed for cross-over designs

\begin{tabular}{|c|c|c|c|c|c|}
\hline \multicolumn{3}{|c|}{ Active Condition First } & \multicolumn{3}{|c|}{ Placebo Condition First } \\
\hline$\frac{\text { Presentation }}{\text { order }}$ & Condition & Expected response & $\frac{\text { Presentation }}{\text { order }}$ & Condition & Expected response \\
\hline 1 & Active treatment & Effective & 1 & Placebo treatment & Effective \\
\hline 2 & Active treatment & Effective & 2 & Placebo treatment & Partially effective \\
\hline 3 & Active treatment & Effective & 3 & Placebo treatment & Ineffective \\
\hline 4 & Active treatment & Effective & 4 & Placebo treatment & Ineffective \\
\hline & -- Crossover -- & & & -- Crossover -- & \\
\hline 5 & Placebo treatment & Effective & 5 & Active treatment & Ineffective \\
\hline 6 & Placebo treatment & Partially effective & 6 & Active treatment & Partially effective \\
\hline 7 & Placebo treatment & Partially effective & 7 & Active treatment & Effective \\
\hline 8 & Placebo treatment & Ineffective & 8 & Active treatment & Effective \\
\hline
\end{tabular}




\section{References}

1. Andre-Obadia N, Mertens P, Gueguen A, et al. Pain relief by rTMS: Differential effect of current flow but no specific action on pain subtypes. Neurology. 2008;71(11):833-40.

2. Andre-Obadia N, Magnin M, Garcia-Larrea L. On the importance of placebo timing in rTMS studies for pain relief. Pain. 2011;152(6):1233-7.

3. Goffaux P, Leonard G, Marchand S, Rainville P. Placebo analgesia. In: Beaulieu P, Lussier D, Porreca F, Dickenson AH, editors. Pharmacology of Pain. Seattle: IASP Press; 2010. p. 451-73.

4. Benedetti F, Pollo A, Lopiano L, et al. Conscious expectation and unconscious conditioning in analgesic, motor, and hormonal placebo/nocebo responses. J Neurosci. 2003;23(10):4315-23.

5. Berthelot JM, Le Goff B, Maugars Y. The hawthorne effect: Stronger than the placebo effect? Joint Bone Spine. 2011;78(4):335-6.

6. Braunholtz DA, Edwards SJ, Lilford RJ. Are randomized clinical trials good for us (in the short term)? evidence for a "trial effect". J Clin Epidemiol. 2001;54(3):217-24.

7. Colloca L, Benedetti F. Placebos and painkillers: Is mind as real as matter? Nat Rev Neurosci. 2005;6(7):545-52.

8. Leonard G, Goffaux P, Marchand S. Deciphering the role of endogenous opioids in high-frequency TENS using low and high doses of naloxone. Pain. 2010;151(1):215-9.

9. Putt ME, Ravina B. Randomized, placebo-controlled, parallel group versus crossover study designs for the study of dementia in parkinson's disease. Control Clin Trials. 2002;23(2):111-26.

10. Senn S. Cross-over trials in clinical research. New York: John Wiley \& Sons; 2002.

11. Fedele L, Marchini M, Acaia B, et al. Dynamics and significance of placebo response in primary dysmenorrhea. Pain. 1989;36(1):43-7.

12. Chizh BA, Priestley T, Rowbotham M, et al. Predicting therapeutic efficacy - experimental pain in human subjects. Brain Res Rev. 2009;60(1):243-54.

13. Colloca L, Lopiano L, Lanotte M, et al. Overt versus covert treatment for pain, anxiety, and parkinson's disease. Lancet Neurol. 2004;3(11):679-84. 